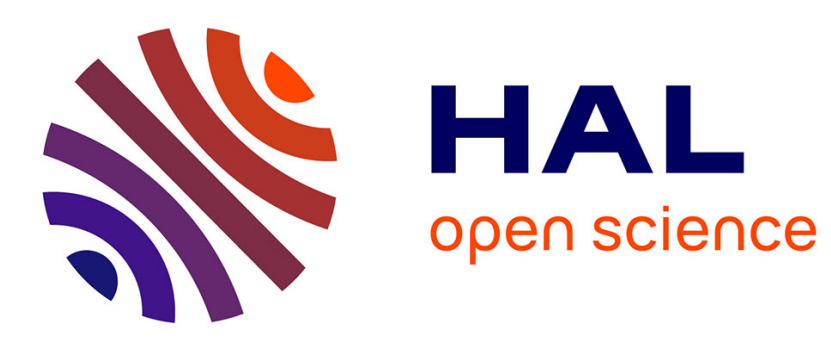

\title{
Two Conformers of N,N-Diethylpropionamide As Observed by Microwave Spectroscopy and Quantum Chemistry
}

Raphaela Kannengiesser, Wolfgang Stahl, Ha Vinh Lam Nguyen

\section{- To cite this version:}

Raphaela Kannengiesser, Wolfgang Stahl, Ha Vinh Lam Nguyen. Two Conformers of N,NDiethylpropionamide As Observed by Microwave Spectroscopy and Quantum Chemistry. Journal of Physical Chemistry A, 2016, 120 (30), pp.5979-5984. 10.1021/acs.jpca.6b03191 . hal-03183110

\section{HAL Id: hal-03183110 https://hal.science/hal-03183110}

Submitted on 26 Mar 2021

HAL is a multi-disciplinary open access archive for the deposit and dissemination of scientific research documents, whether they are published or not. The documents may come from teaching and research institutions in France or abroad, or from public or private research centers.
L'archive ouverte pluridisciplinaire HAL, est destinée au dépôt et à la diffusion de documents scientifiques de niveau recherche, publiés ou non, émanant des établissements d'enseignement et de recherche français ou étrangers, des laboratoires publics ou privés. 


\title{
Two Conformers of N,N-Diethylpropionamide as
}

\section{Observed by Microwave Spectroscopy and}

\section{Quantum Chemistry}

\author{
Raphaela Kannengießer ${ }^{a}$, Wolfgang Stahl ${ }^{a}$, and Ha Vinh Lam Nguyen ${ }^{b^{*}}$

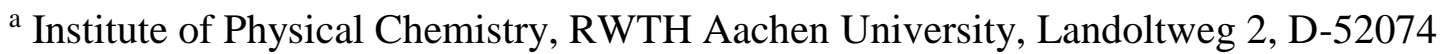 \\ Aachen, Germany \\ ${ }^{\mathrm{b}}$ Laboratoire Interuniversitaire des Systèmes Atmosphériques (LISA), CNRS UMR 7583, \\ Université Paris-Est Créteil, Université Paris Diderot, 61 avenue du Général de Gaulle, F- \\ 94010 Créteil cedex, France
}

\begin{abstract}
The microwave spectra of two conformers of N,N-diethylpropionamide were recorded using a molecular beam Fourier transform microwave spectrometer operating in the frequency range 2-26.5 GHz. Hyperfine splittings arising from the ${ }^{14} \mathrm{~N}$ quadrupole coupling effect were observed and analyzed. Fits using a rigid rotor model with centrifugal distortion correction and first order perturbation approach for the quadrupole coupling yielded highly accurate molecular parameters and standard deviations within the measurement accuracy for both conformers. Complementary quantum chemical calculations were carried out for a conformational analysis and theoretical values of the spectroscopic constants and the ${ }^{14} \mathrm{~N}$ nuclear quadrupole coupling constants.
\end{abstract}




\section{INTRODUCTION}

The structures of amides are of biological and chemical interest, since the peptide linkage $-(\mathrm{C}=\mathrm{O}) \mathrm{NH}-$ present in amides is a characteristic element in proteins. In the condensed phase, extensive hydrogen bonded networks that are formed by the proton accepting carbonyl oxygen atom $-(\mathrm{C}=\mathbf{O})$ and the amminic hydrogen atom(s) NH- may obscure the genuine structural and conformational properties. Gas phase studies are thus preferred to obtain the best possible insight in the unperturbed structures of amides. Microwave spectroscopy with its high resolution is a state-of-the-art experimental method for this purpose. Unfortunately, amides often possess low vapor pressures and tend to decompose upon heating. These properties have made their gas phase investigations less accessible than for other molecular classes, e.g. esters, resulting in a relatively limited literature about this class of compounds.

The simplest amide, formamide, $\mathrm{H}(\mathrm{C}=\mathrm{O}) \mathrm{NH}_{2}$, has only one conformer, which is completely planar. ${ }^{1}$ Several conformations are possible when one or more hydrogen atoms are substituted by ethyl group(s). Substituting the formyl hydrogen atom $\mathbf{H}(\mathrm{C}=\mathrm{O})$ results in propionamide $\mathrm{CH}_{3} \mathrm{CH}_{2}(\mathrm{C}=\mathrm{O}) \mathrm{NH}_{2}$, whose gas phase structure has been thoroughly studied by Marstokk et $a .^{2}$ This investigation has revealed a number of interesting and unexpected findings. The complementary quantum chemical calculations at the MP2/6-311++G(d,p) level of theory predict a non-planar conformer, in which the ethyl group is tilted out of the $(\mathrm{C}=\mathrm{O}) \mathrm{NH}_{2}$ plane by an angle of $23.35^{\circ}$. The experimental results by microwave spectroscopy on the other hand indicate only one observed conformer with an effective $\mathrm{C}_{\mathrm{s}}$ equilibrium conformation. This type of symmetry breaking where the propionyl ethyl group $\mathrm{CH}_{3} \mathrm{CH}_{2}(\mathrm{C}=\mathrm{O})$ - is calculated to be out of the carbonyl plane is a frequent result in quantum chemical calculations at the MP2/6-311++G(d,p) level of theory. For example, a tilt angle of $8.4^{\circ}$ out of the $\mathrm{C}-(\mathrm{C}=\mathrm{O})-\mathrm{C}$ plane is predicted for the ethyl group in ethyl methyl ketone. ${ }^{3}$ A similar tilt angle of about $12^{\circ}$ is found for each ethyl group in diethyl ketone. ${ }^{4}$ The tilt angles are greater, when one or both 
$\mathrm{CH}_{2}$ hydrogen atoms are substituted by methyl groups. For the isopropyl group in methyl isobutyl ketone ${ }^{5}$ and the tert-butyl group in pinacolone, ${ }^{6}$ ab initio calculations predict tilt angles of approximately $20^{\circ}$ and $16^{\circ}$, respectively, which are close to the value reported above for propionamide.

Substituting one of the hydrogen atoms in the amide group $-(\mathrm{C}=\mathrm{O}) \mathrm{NH}_{2}$ by an ethyl group results in N-ethylformamide, $\mathrm{H}(\mathrm{C}=\mathrm{O})(\mathrm{NH}) \mathrm{CH}_{2} \mathrm{CH}_{3}$. Its gas phase structure has been reported in the literature. ${ }^{7}$ Only one conformer is present in the microwave spectrum, in which the ethyl group is not located in the $\mathrm{H}(\mathrm{C}=\mathrm{O}) \mathrm{NH}$ plane, but bends out by an angle of $82.17(19)^{\circ}$. This angle is determined experimentally from the $r_{s}$ structure obtained by isotopic substitutions. A similar tilt angle of the ethyl group is found for the observed conformer in the microwave spectrum of N-ethylacetamide. ${ }^{8}$

By substituting both hydrogen atoms in the amide group by ethyl groups, N,N-diethylamides are obtained. Only one example for this type of molecule is available in the literature, namely the N,N-diethylacetamide, $\mathrm{CH}_{3}(\mathrm{C}=\mathrm{O}) \mathrm{N}\left(\mathrm{CH}_{2} \mathrm{CH}_{3}\right)_{2} \cdot{ }^{9}$ Two conformers exist in the microwave spectrum under molecular beam conditions, both have the two ethyl groups bending out of the $\mathrm{C}-(\mathrm{C}=\mathrm{O}) \mathrm{N}$ plane. In the more stable conformer, the ethyl groups are out of the carbonyl plane in opposite directions, whereas the other conformer has the ethyl groups bending in the same direction. Obviously in this case, the steric effect mainly governs the stability of different the conformers.

In the present work, we extend the investigations on amides to N,N-diethylpropionamide (DEPA), where all the three hydrogen atoms in formamide are substituted by ethyl groups. The conformational analysis is carried out by microwave spectroscopy supplemented by quantum chemical calculations, aiming to answer the following questions: (i) will only one or several conformers of DEPA be observed in the microwave spectrum, (ii) which orientations of the three ethyl groups will be present in the most stable conformer, and in particular (iii) 
will a conformer with a planar propionyl group exist and if yes, is the propionyl group completely planar?

Beside the interesting conformational landscape, DEPA shows ${ }^{14} \mathrm{~N}$ nuclear quadrupole coupling. This effect is caused by the interaction of the nuclear quadrupole moment with the electric field gradient (EFG) at the site of the nucleus, which originates from the surrounding electron cloud. In atoms, the electronic charge distribution is spherical, and the EFG is zero. However, in a molecule, the atomic electron clouds are distorted due to their contribution to the chemical bonds, and usually a non-zero EFG results. Therefore, the nuclear quadrupole coupling is closely related to the character of the chemical bond, which has been worked out quantitatively by Townes and Dailey. ${ }^{10}$ Hyperfine splittings arising from this effect are from some tens of $\mathrm{kHz}$ up to $1 \mathrm{MHz}$ and can be resolved with the molecular beam Fourier transform technique. The ${ }^{14} \mathrm{~N}$ Nuclear Quadrupole Coupling Constants (NQCCs) of DEPA will be determined and compared to those of other propionamides and acetamides.

\section{THEORETICAL}

Reasonable starting values of molecular parameters such as rotational constants and ${ }^{14} \mathrm{~N}$ quadrupole coupling constants are important for spectral assignment, because reliable predictions of the spectrum considerably simplify the assignment process. Quantum chemistry is a powerful tool for this purpose. The GAUSSIAN 09 suite of programs ${ }^{11}$ was employed for all calculations in this work.

Conformational preferences. For a conformational analysis, $6^{4}=1296$ starting geometries were automatically generated by a program varying the four dihedral angles $\angle\left(\mathrm{N}_{1}, \mathrm{C}_{4}, \mathrm{C}_{6}, \mathrm{C}_{7}\right)$, $\angle\left(\mathrm{C}_{4}, \mathrm{~N}_{1}, \mathrm{C}_{2}, \mathrm{C}_{15}\right), \angle\left(\mathrm{C}_{4}, \mathrm{~N}_{1}, \mathrm{C}_{3}, \mathrm{C}_{11}\right)$, and $\angle\left(\mathrm{O}_{5}, \mathrm{C}_{4}, \mathrm{~N}_{1}, \mathrm{C}_{3}\right)$ (for atom numbering see Figure 1) over a grid of $60^{\circ}$, and fully optimized at the MP2/6-311++G(d,p) level of theory. The 
optimizations yielded ten conformers, which were confirmed as minima on the potential energy surface by frequency calculations. The rotational constants, dipole moment components, and energies relative to that of the lowest energy conformer are given in Table 1.

In order to determine the energetically most favorable conformers, not only the calculated electronic energies but also the zero point energies (ZPE) must be considered. We took the ZPE correction into account for all method/basis set combinations except for the MP2/ccpVTZ and MP2/6-311++G(3df,2pd) levels, where our computational resources did not allow for frequency calculations. Therefore, these two levels of theory are valuable to obtain accurate rotational constants but they cannot be used to estimate the relative abundances of different conformers in the molecular beam.

The structures of the three most energetically favorable conformers are given in Figure 1; the nuclear coordinates in the principal axis system are available in Tables S-1 in the Supporting Information. They differ by up to $2 \mathrm{~kJ} \cdot \mathrm{mol}^{-1}$ in energy. On the other hand, their calculated total dipole moments have similar values of approximately 4.1 Debye. All of them might be present in the experimental spectrum under our molecular beam conditions using helium as carrier gas, because the conformational cooling is not as effective as with neon or argon and higher energy conformers could be still populated. The other seven conformers are much higher in energy and are presumably not observable. The lowest energy conformer, which is henceforth called 0PM, has the propionyl ethyl group almost located on the $(\mathrm{C}=\mathrm{O}) \mathrm{N}$ plane (marked with 0) and the other two ethyl groups bending into opposite sides of this plane (marked with plus/P and minus/M). In the same manner, the other two conformers are named MMP and OMM.

The MP2/6-311++G(d,p) level of theory predicts that conformer MMP is $0.7 \mathrm{~kJ} \cdot \mathrm{mol}^{-1}$ lower in energy than conformer OMM. Re-optimizations using other basis sets including diffuse 
functions yielded the same results. On the other hand, if the diffuse functions are omitted, or a correlation consistent basis set is used, OMM becomes more stable than MMP, which are also the results of HF and DFT calculations (see Table 2). This disagreement in energy calculations at different levels of theory challenges the assignment process, as will be revisited in the sections on Microwave Spectrum and Discussion.

${ }^{14} \mathrm{~N}$ quadrupole coupling constants. Because of the ${ }^{14} \mathrm{~N}$ nucleus with a nuclear spin of $I=1$, all rotational transitions of DEPA split into several quadrupole components. These hyperfine splittings depend on the NQCCs. From the optimized structure at the MP2/6-311++G(d,p) level, we carried out a single point EFG calculation at the B3PW91/6-311+(df,pd) level of theory using a calibration factor of $\mathrm{eQ} / \mathrm{h}=4.5586(40) \mathrm{MHz}$ a.u. ${ }^{-1}$ given by Bailey. ${ }^{12,13}$ The calculation results in nuclear quadrupole coupling tensors with the diagonal elements $\chi_{a a}=1.9490 \mathrm{MHz}, \chi_{b b}=1.1471 \mathrm{MHz}$, and $\chi_{c c}=-3.0961 \mathrm{MHz}$, as well as the off-diagonal elements $\chi_{a b}=-0.2437 \mathrm{MHz}, \chi_{a c}=-0.5440 \mathrm{MHz}$, and $\chi_{b c}=2.6623 \mathrm{MHz}$ for conformer OPM. For conformer OMM values of $\chi_{a a}=1.8180 \mathrm{MHz}, \chi_{b b}=2.3961 \mathrm{MHz}, \chi_{c c}=-4.2142$ $\mathrm{MHz}, \chi_{a b}=-0.0566 \mathrm{MHz}, \chi_{a c}=-1.0620 \mathrm{MHz}$, and $\chi_{b c}=-0.3363 \mathrm{MHz}$ were found. The ${ }^{14} \mathrm{~N}$ NQCCs calculated with this method match the experimental values almost exactly in many of our previous investigations on nitrogen containing molecules. ${ }^{8,14,15}$

\section{MICROWAVE SPECTRUM}

Experimental. Commercially available DEPA from TCI Europe, Zwijndrecht, Belgium, with a stated purity of $98 \%$ was used. All spectra were recorded using a molecular beam Fouriertransform microwave spectrometer described previously in Ref. [16]. Because of the low vapor pressure of DEPA, a pipe-cleaner as carrier material was soaked with the substance and placed upstream the nozzle. Helium, which was used as a carrier gas, was flown over the 
sample, and the gas mixture was expanded into a vacuum chamber at a total pressure of 150 to $200 \mathrm{kPa}$.

A broadband scan in the frequency range $8.5-16.0 \mathrm{GHz}$, where overlapping spectra with a step width of $250 \mathrm{kHz}$ and 50 co-added decays were automatically taken, revealed a rich and complex spectrum. All lines in the scan have line widths of a few $\mathrm{MHz}$ due to quadrupole interaction. A portion of $7.5 \mathrm{GHz}$ of the scan is given in Figure 2; a portion of $50 \mathrm{MHz}$ in Figure 3. All lines were subsequently remeasured at higher resolution with an intrinsic experimental line width of $2 \mathrm{kHz}$ for isolated lines, ${ }^{17}$ indicating completely resolved hyperfine splittings arise from the spin $I=1$ of the nitrogen nucleus. Figure 4 shows a typical spectrum of DEPA measured at high resolution. The line widths are $14-30 \mathrm{kHz}$, corresponding to a measurement accuracy of $2 \mathrm{kHz}$.

Conformer 0PM. We started the assignment with the spectrum of the lowest energy conformer 0PM. As a first step, we neglected quadrupole coupling and used a rigid-rotor program to calculate the theoretical spectra. The most intense lines in the spectrum were readily assigned to conformer OPM by comparing their frequencies with the predicted spectrum. Thereafter, hyperfine splittings were calculated using the NQCCs given in section ${ }^{14} \mathrm{~N}$ Quadrupole Coupling Constants. In total, 91 rotational transitions with 368 hyperfine components were assigned and fitted with the program XIAM, ${ }^{18}$ which is based in our mode of operation on the Hamiltonian $\mathbf{H}=\mathbf{H}_{\mathrm{rot}}+\mathbf{H}_{\mathrm{CD}}+\mathbf{H}_{\mathrm{NQ}}$, where $\mathbf{H}_{\text {rot }}$ is the usual rigid rotor Hamiltonian, $\mathbf{H}_{\mathrm{CD}}$ the centrifugal distortion contribution (in this case in the form of Watson's S reduction), and $\mathbf{H}_{\mathrm{NQ}}$ a scalar product of the second rank field gradient tensor $\mathbf{V}^{(2)}$ and the second rank quadrupole tensor $\mathbf{Q}^{(2)} \cdot{ }^{19} \mathbf{H}_{\mathrm{NQ}}$ was treated in first order, i.e. only matrix elements diagonal in $J$ were considered. The standard deviation of this fit is $2.2 \mathrm{kHz}$, close the measurement accuracy. 
Conformer OMM. After conformer OPM was assigned, many weaker lines remained in the spectrum, which might belong to conformer MMP and/or 0MM. By trial and error, 56 lines in the broadband scan could be assigned to conformer 0MM. They contain 220 quadrupole components and were fitted to a standard deviation of $1.7 \mathrm{kHz}$. The molecular parameters of both conformers $\mathrm{OPM}$ and $\mathrm{OMM}$ are summarized in Table 3; the transition frequencies are collected in Table S-2 and S-3, respectively, in the Supporting Information. The correlation matrices of both fits are given in Table S-4 and S-5.

It should be noted that after excluding all transitions belonging to conformer OPM and 0MM, some weaker lines remain in the spectrum. Hard efforts to assign these lines to conformer MMP did not lead to success. We thus conclude that only conformers OPM and OMM are present in our spectrum, whereby conformer $0 \mathrm{PM}$ is lower in energy than conformer 0MM. Splittings due to internal rotation of the three ethyl methyl groups were not observed for both conformers. The barriers of internal rotation of such groups are usually higher than $1000 \mathrm{~cm}^{-1}$, and the resulting splittings are not resolvable with our spectrometer. ${ }^{9,14,20}$

\section{DISCUSSION}

The microwave spectra of two conformers of DEPA, called OPM and OMM, with 368 and 220 hyperfine transitions were assigned and fitted with excellent standard deviations of $2.2 \mathrm{kHz}$ and $1.7 \mathrm{kHz}$, respectively. A rigid rotor model with centrifugal distortion correction and a first order perturbation approach for ${ }^{14} \mathrm{~N}$ nuclear quadrupole coupling was sufficient to reproduce the experimental spectra to measurement accuracy of $2 \mathrm{kHz}$. All parameters were determined with very high accuracy as shown in Table 3.

As mentioned in the section on conformational preferences, calculations at different levels of theory point out that the MP2 method in combination with diffuse functions calculate 
conformer MMP to be lower in energy than conformer 0MP (see Table 2). The experimental results clearly show the contrary. This combination, which includes our most frequently used MP2/6-311++G(d,p) level of theory, might not be suitable for calculating the conformational energies of molecules like DEPA, at least for our experimental conditions. On the other hand, the rotational constants predicted for both conformers at the MP2/6-311++G(d,p) level are very close to the experimental values. The differences are within $0.5 \%$, which was extremely helpful for the assignment process. The centrifugal distortion constants agree reasonably with the experimental values.

The propionyl ethyl group possesses a dihedral angle $\angle\left(\mathrm{N}_{1}, \mathrm{C}_{4}, \mathrm{C}_{6}, \mathrm{C}_{7}\right)$ of $-168.6^{\circ}$ and $-171.0^{\circ}$ for conformer OPM and OMM, respectively. Correspondingly, the ethyl group is slightly tilted out of the $(\mathrm{C}=\mathrm{O}) \mathrm{N}$ plane by a respective angle of $11.4^{\circ}$ and $9.0^{\circ}$. This is almost the same as the angle found for the propionyl ethyl group in ethyl methyl ketone $\left(8.4^{\circ}\right)^{3}$ and diethyl ketone $\left(12^{\circ}\right){ }^{4}$ The orientations of the ethyl groups attached to the nitrogen atom are the same as those in $\mathrm{N}, \mathrm{N}$-diethylacetamide, i.e. in the more stable conformer, they point in opposite directions, whereas in the other conformer they are oriented in the same direction. In OPM, the dihedral angles $\angle\left(\mathrm{C}_{4}, \mathrm{~N}_{1}, \mathrm{C}_{3}, \mathrm{C}_{11}\right)$ and $\angle\left(\mathrm{C}_{4}, \mathrm{~N}_{1}, \mathrm{C}_{2}, \mathrm{C}_{15}\right)$ are $98.2^{\circ}$ and $84.3^{\circ}$, respectively. The respective angles of conformers I of $\mathrm{N}, \mathrm{N}$-diethylacetamide ${ }^{9}$ are $98.0^{\circ}$ and $84.8^{\circ}$, which are essentially the same. In $0 \mathrm{MM}$, we found that $\angle\left(\mathrm{C}_{4}, \mathrm{~N}_{1}, \mathrm{C}_{3}, \mathrm{C}_{11}\right)=107.7^{\circ}$ and $\angle\left(\mathrm{C}_{4}, \mathrm{~N}_{1}, \mathrm{C}_{2}, \mathrm{C}_{15}\right)$ $=-75.9^{\circ}$, which are also very close to the values of conformer II of N,N-diethylacetamide ${ }^{9}$ $\left(108.5^{\circ}\right.$ and $-75.8^{\circ}$, respectively). Obviously, the steric effect has the largest influence on the stability of the conformers of DEPA.

The experimental NQCCs are as expected in almost exact agreement with the values calculated with the method given in the section on ${ }^{14} \mathrm{~N}$ quadrupole coupling constants. The NQCC $z$-principal axis components $\chi_{\mathrm{zz}}$ of conformers OPM and OMM are similar (see Table 
4), i.e. the orientations of the ethyl groups do not affect the electronic environment at the site of the nitrogen nucleus.

Comparing the $\chi_{z z}$ values of amides related to DEPA (Table 4), we found that (i) $\chi_{z z}$ gradually increases from $3.8510(11) \mathrm{MHz}$ in formamide to $4.455(28) \mathrm{MHz}$ in DEPA; (ii) different conformers possess the same $\chi_{\mathrm{zz}}$ values (within the errors); (iii) $\chi_{\mathrm{zz}}$ becomes larger with an increase in the $+\mathrm{I}$ effect of the alkyl substituents $\left(\mathrm{H}-<\mathrm{CH}_{3}-<\mathrm{CH}_{3} \mathrm{CH}_{2}-\right)$; and (iv) different molecules with the same alkyl substituents attached to the $(\mathrm{C}=\mathrm{O})-\mathrm{N}$ frame have essentially the same $\chi_{\mathrm{zz}}$ values, independent of the place of substitution.

\section{CONCLUSIONS}

The microwave spectra from 8.5 to $16.0 \mathrm{GHz}$ of two conformers of N,N-diethylpropionamide showing ${ }^{14} \mathrm{~N}$ quadrupole coupling were assigned and fitted using the program XIAM. The standard deviations are within the measurement accuracy of $2 \mathrm{kHz}$. In both conformers, the propionyl ethyl group is located in (or almost in) the $(\mathrm{C}=\mathrm{O}) \mathrm{N}$ plane, while the other two ethyl groups are tilted out. In the more stable conformer, the latter ethyl groups are oriented in opposite directions, whereas in the other conformer they point in the same direction. The steric effect is clearly the reason for this observation.

The molecular parameters could be determined with very high accuracy. Calculations at the MP2/6-311++G(d,p) level yielded excellent values for the rotational constants and centrifugal distortion constants. In combination with the B3PW91/6-311+(df,pd) single point EFG calculation and the calibration factor of $\mathrm{eQ} / \mathrm{h}=4.5586(40) \mathrm{MHz}$ a.u. ${ }^{-1}$, this level of theory results in ${ }^{14} \mathrm{~N}$ NQCCs in almost exact agreement with the experimental values. However, energy calculations at this level turn out to be unreliable. 
Comparison of the ${ }^{14} \mathrm{~N}$ NQCC $z$-principal axis component $\chi_{z z}$ of N,N-diethylpropionamide and related amides indicates that the increasing $+\mathrm{I}$ effect of the alkyl substituents at the $(\mathrm{C}=\mathrm{O})-\mathrm{N}$ frame slightly increase the $\chi_{z z}$ value.

\section{ASSOCIATED CONTENT}

Supporting Information. Cartesian coordinates of the conformers OPM, OMM and MMP, lists of fitted frequencies along with their residuals of conformer OPM and OMM, correlation matrices of both fits. This material is available free of charge via the Internet at http://pubs.acs.org.

\section{AUTHOR INFORMATION}

\section{Corresponding Author}

* Email: lam.nguyen@ lisa.u-pec.fr, Tel.: +33 145176548.

\section{ACKNOWLEDGMENT}

We thank the Land Nordrhein-Westfalen for funds, the IT Center of the RWTH Aachen University for free computer time, and M.Sc. V. Van for his support on the conformational search. R. K. thanks the graduate fond of the RWTH Aachen University for a Ph.D. fellowship. 


\section{REFERENCES}

(1) Kryvda, A. V.; Gerasimov, V. G.; Dyubko, S. F.; Alekseev, E. A.; Motiyenko, R. A. New Measurements of the Microwave Spectrum of Formamide. J. Mol. Spectrosc. 2009, 254, 28-32.

(2) Marstokk, K-M.; Møllendal, H.; Samdal, S. Microwave Spectrum, Conformation, ab initio Calculations, Barrier to Internal Rotation and Dipole Moment of Propionamide. J. Mol. Struct. 1996, 376, 11-24.

(3) Nguyen, H. V. L.; Van, V.; Stahl, W.; Kleiner, I. The Effects of Two Internal Rotations in the Microwave Spectrum of Ethyl Methyl Ketone. J. Chem. Phys. 2014, $140,214303$.

(4) Nguyen, H. V. L.; Stahl, W. The Rotational Spectrum of Diethyl Ketone ChemPhysChem 2011, 12, 1900-1905.

(5) Zhao, Y.; Stahl, W.; Nguyen, H. V. L. Ketone Physics - Structure, Conformations, and Dynamics of Methyl Isobutyl Ketone Explored by Microwave Spectroscopy and Quantum Chemical Calculations. Chem. Phys. Lett. 2012, 545, 9-13.

(6) Zhao, Y.; Nguyen, H. V. L.; Stahl, W.; Hougen, J. T. Unusual Internal Rotation Coupling in the Microwave Spectrum of Pinacolone. J. Mol. Spectrosc. 2015, 318, 91-100.

(7) Ohba, K.; Usami, T.; Kawashima, Y.; Hirota, E. Fourier Transform Microwave Spectra and Ab Initio Calculation of N-Ethylformamide. J. Mol. Struct. 2005, 744, 815-819. 
(8) Kannengießer, R.; Lach, M. J.; Stahl, W.; Nguyen, H. V. L. Acetyl Methyl Torsion in N-Ethylacetamide: A Challenge for Microwave Spectroscopy and Quantum Chemistry. ChemPhysChem. 2015, 16, 1906-1911.

(9) Kannengießer, R.; Klahm, S.; Nguyen, H. V. L.; Lüchow, A.; Stahl, W. The Effects of Methyl Internal Rotation and ${ }^{14} \mathrm{~N}$ Quadrupole Coupling in the Microwave Spectra of Two Conformers of N,N-Diethylacetamide. J. Chem. Phys. 2014, 141, 204308.

(10) Townes, C. T.; Dailey, B. P. Determination of Electronic Structure of Molecules from Nuclear Quadrupole Effects. J. Chem. Phys. 1949, 17, 782-796.

(11) Frisch, M. J.; Trucks, G. W.; Schlegel, H. B.; Scuseria, G. E.; Robb, M. A.; Cheeseman, J. R.; Scalmani, G.; Barone, V.; Mennucci, B.; Petersson, G. A. et al. Gaussian 09, Revision A.02, Gaussian, Inc.; Wallingford CT, 2009.

(12) Bailey, W. C. DFT and HF-DFT Calculations of ${ }^{14} \mathrm{~N}$ Quadrupole Coupling Constants in Molecules. Chem. Phys. 2000, 252, 57-66.

(13) Bailey, W. C. Calculation of Nuclear Quadrupole Coupling Constants in Gaseous State Molecules, 2016, http://nqcc.wcbailey.net/Publ_N.html.

(14) Nguyen, H. V. L.; Kannengießer, R.; Stahl, W. Microwave Survey of the Conformational Landscape Exhibited by the Propeller Molecule Triethyl Amine. Phys. Chem. Chem. Phys. 2012, 14, 11753-11758.

(15) Kannengießer, R.; Stahl, W.; Nguyen, H. V. L.; Bailey W. C. ${ }^{14}$ N Quadrupole Coupling in the Microwave Spectra of N-Vinylformamide. J. Mol. Spectrosc. 2015, $317,50-53$. 
(16) Grabow, J. U.; Stahl, W.; Dreizler, H. A Multioctave Coaxially Oriented Beam-Resonator Arrangement Fourier-Transform Microwave Spectrometer. Rev. Sci. Instrum. 1996, 67, 4072-4084.

(17) Grabow, J.-U.; Stahl, W. A Pulsed Molecular Beam Microwave Fourier Transform Spectrometer with Parallel Molecular Beam and Resonator Axes. Z. Naturforsch. 1990, 45a, 1043-1044.

(18) Hartwig, H.; Dreizler, H. The Microwave Spectrum of trans-2,3-Dimethyloxirane in Torsional Excited States. Z. Naturforsch. 1996, 51a, 923-932.

(19) Gordy, W.; Cook, R. L. Microwave Molecular Spectra. John Wiley \& Sons, New York 1984, $3^{\text {rd }}$ edition

(20) Fliege, E.; Dreizler, H.; Demaison, J.; Boucher, D.; Burie, J.; Dubrulle, A. Centrifugal Distortion and Internal Rotation Analysis of the Microwave Spectrum of Ethyl Fluoride. J. Chem. Phys. 1983, 78, 3541-3544.

(21) Kirchhoff, W. H.; Johnson, D. R. An Investigation of Centrifugal Distortion in the Microwave Spectrum of Formamide. J. Mol. Spectrosc. 1973, 45, 159-165.

(22) Mescheryakov, A. A.; Alekseev, E. A.; Ilyushin, V.; Motiyenko, R. A., Margulès, L.; Lovas, F. J. Millimeter and Submillimeter Wave Spectra of N-Methyl Formamide and Propionamide. 69th International Symposium on Molecular Spectroscopy 2014.

(23) Kawashima, Y.; Suenram, R. D.; Hirota, E. Dynamical Structure of Peptide Molecules: Fourier Transform Microwave Spectroscopy of N-Methylpropionamide. $J$. Mol. Spectrosc. 2003, 219, 105-118. 


\section{FIGURES}<smiles>CCC(=O)N(CC)CC</smiles>

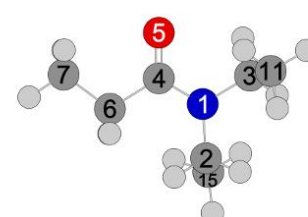

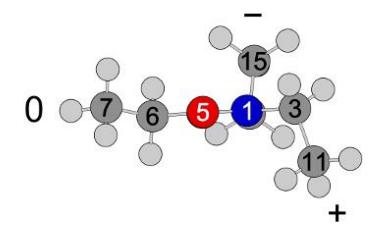<smiles>CCC(=O)N(CC)CC</smiles>
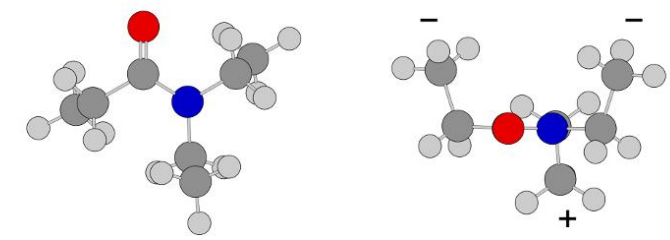<smiles>CCC(=O)N(CC)CC</smiles>
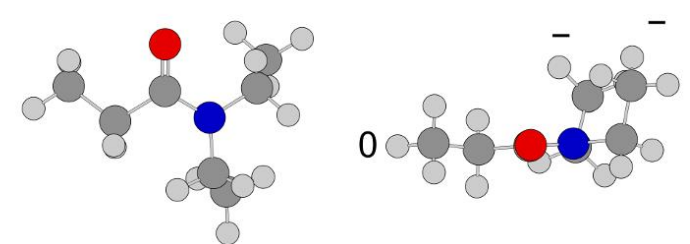

Figure 1. Optimized geometries of the three most stable conformers of $\mathrm{N}, \mathrm{N}$-diethylpropionamide. Ethyl groups, which are out of the $(\mathrm{C}=\mathrm{O}) \mathrm{N}$ plane, are characterized with $\mathrm{P}(+)$ or M (-); ethyl groups located in or almost in this plane with 0 . Left hand side: Natta projections, middle: front view, right hand side: view along the $\mathrm{O}_{5}-\mathrm{C}_{4}$ bond. 


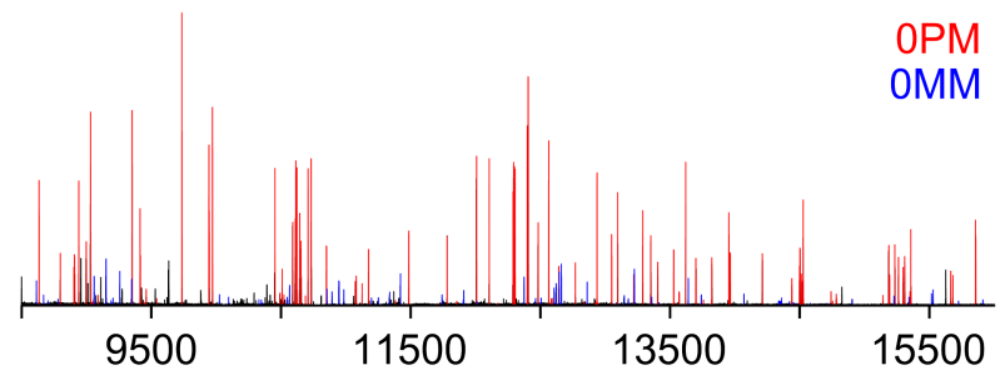

Figure 2. The broadband scan from $8500 \mathrm{MHz}$ to $16000 \mathrm{MHz}$ of N,N-diethylpropionamide showing that (i) the transitions of conformer OPM are much more intense than those of conformer 0MM and (ii) only some weak unassigned lines are present in the spectrum.

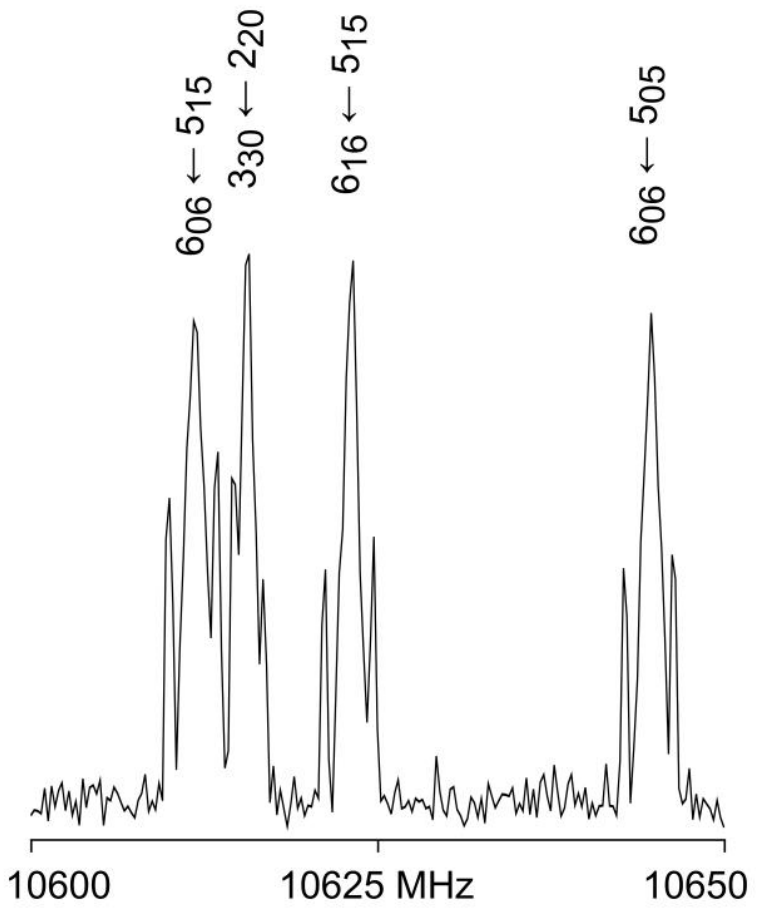

Figure 3. A portion of $50 \mathrm{MHz}$ of the broadband scan of $\mathrm{N}, \mathrm{N}$-diethylpropionamide. All indicated transitions belong to conformer $0 \mathrm{PM}$ and are marked with their rigid rotor quantum numbers $J, K_{a}, K_{c}$. The lines are broadened or split because of the quadrupole coupling arise from the ${ }^{14} \mathrm{~N}$ nucleus. 


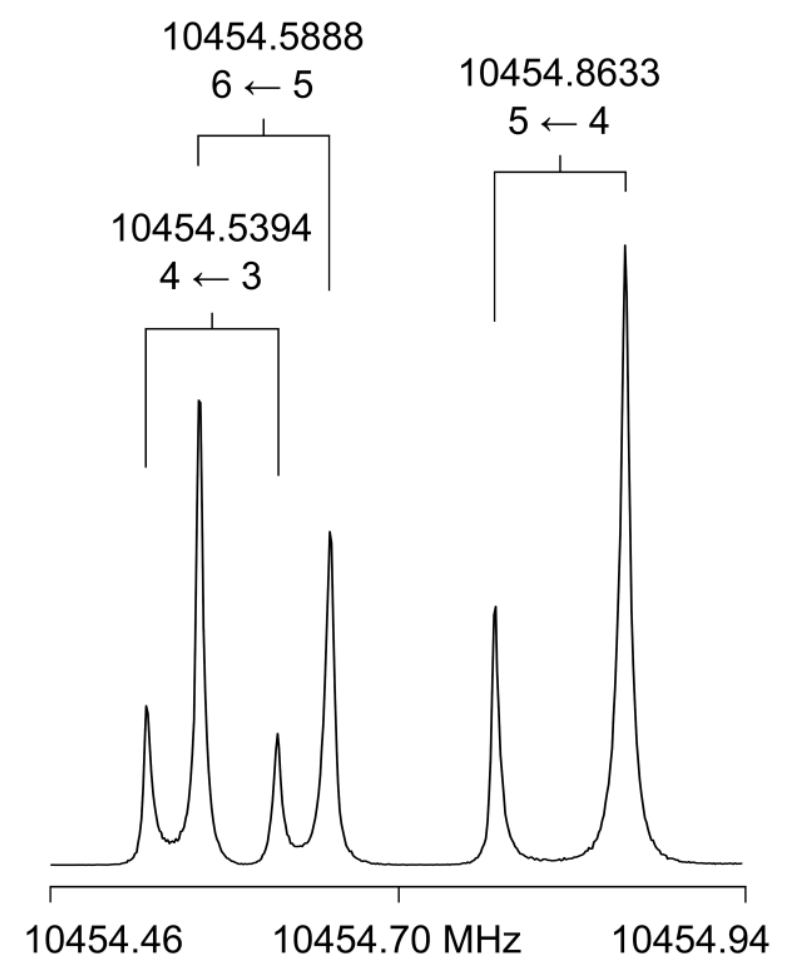

Figure 4. A typical spectrum of the $5_{14} \leftarrow 4_{13}$ transition of conformer OPM of DEPA with three quadrupole components given by F' $\leftarrow$ F. Brackets indicate the Doppler doublets. For this spectrum, 1000 decays were co-added. 


\section{TABLES}

Table 1. Energies with zero-point corrections in Hartree, relative energies in $\mathrm{kJ} \cdot \mathrm{mol}^{-1}$, rotational constants in $\mathrm{MHz}$ and dipole moment components in $\mathrm{D}$ of the ten conformers of $\mathrm{N}, \mathrm{N}$-diethylpropionamide calculated at the MP2/6-311++G(d,p) level of theory. All values refer to the principal axis system.

\begin{tabular}{lcccccccc}
\hline & $\mathrm{E}_{\mathrm{MP} 2+Z \mathrm{ZPE}}$ & $\mathrm{E}_{\text {rel }}$ & $A$ & $B$ & $C$ & $\left|\mu_{\mathrm{a}}\right|$ & $\left|\mu_{\mathrm{b}}\right|$ & $\left|\boldsymbol{\mu}_{\mathrm{c}}\right|$ \\
\hline $\mathbf{0 P M}$ & $\mathbf{- 4 0 4 . 4 5 6 4 5 7}$ & $\mathbf{0 . 0 0}$ & $\mathbf{1 9 1 5 . 4}$ & $\mathbf{1 2 2 0 . 8}$ & $\mathbf{8 3 3 . 7}$ & $\mathbf{1 . 6 2 5}$ & $\mathbf{3 . 5 1 4}$ & $\mathbf{1 . 4 2 2}$ \\
MMP & -404.455836 & 1.63 & 1698.7 & 1344.2 & 948.8 & 0.826 & 3.695 & 1.927 \\
$\mathbf{0 M M}$ & $\mathbf{- 4 0 4 . 4 5 5 5 7 5}$ & $\mathbf{2 . 3 2}$ & $\mathbf{2 0 7 1 . 2}$ & $\mathbf{1 1 7 7 . 1}$ & $\mathbf{8 5 6 . 3}$ & $\mathbf{1 . 7 7 5}$ & $\mathbf{3 . 6 7 6}$ & $\mathbf{0 . 3 3 1}$ \\
PMM & -404.454657 & 4.73 & 1989.1 & 1268.8 & 892.3 & 0.504 & 4.158 & 0.529 \\
PPP & -404.454284 & 5.71 & 1825.3 & 1371.9 & 961.3 & 0.564 & 4.166 & 0.576 \\
MPM & -404.454005 & 6.44 & 1857.5 & 1361.2 & 890.8 & 0.440 & 3.935 & 1.491 \\
MMM & -404.453733 & 7.15 & 1929.1 & 1283.3 & 947.3 & 0.963 & 3.957 & 0.602 \\
OP0 & -404.453661 & 7.34 & 2296.9 & 1076.4 & 811.0 & 2.077 & 3.259 & 1.109 \\
OPP & -404.453597 & 7.51 & 2125.6 & 1184.8 & 866.8 & 1.448 & 3.707 & 0.943 \\
P0M & -404.452639 & 10.02 & 2005.6 & 1157.6 & 940.8 & 1.293 & 3.689 & 1.480 \\
\hline
\end{tabular}


Table 2. Sum of the electronic and zero-point energies of the three most stable conformers of DEPA calculated at different levels of theory. Energies relative to the lowest energy conformer $0 \mathrm{PM}$ are given in $\mathrm{kJ} \cdot \mathrm{mol}^{-1}$, absolute energies of conformer OPM in Hartree.

\begin{tabular}{lcccr}
\hline & \multicolumn{1}{c}{$\boldsymbol{E}_{\boldsymbol{O P M}}$} & $\boldsymbol{E}_{\boldsymbol{O M} \boldsymbol{M}}$ & $\boldsymbol{E}_{\boldsymbol{M M P}}$ & $\boldsymbol{\Delta E}^{\boldsymbol{a}}$ \\
\hline MP2 & & & & \\
cc-pVTZ & -404.935066 & 2.17 & 2.24 & 0.07 \\
6-311++G(3df,2pd) & -404.940760 & 2.33 & 1.12 & -1.21 \\
6-311++G(d,p) & -404.456455 & 2.32 & 1.63 & -0.69 \\
6-311+G(d,p) & -404.455308 & 2.29 & 1.74 & -0.55 \\
6-311G(d,p) & -404.443380 & 2.40 & 3.67 & 1.27 \\
6-31++G(d,p) & -404.314998 & 2.66 & 1.76 & -0.91 \\
6-31+G(d,p) & -404.312833 & 2.67 & 1.85 & -0.83 \\
6-31G(d,p) & -404.291421 & 2.46 & 4.48 & 2.03 \\
B3LYP & & & & \\
cc-pVTZ & -405.717289 & 3.15 & 4.30 & 1.16 \\
6-311++G(3df,2pd) & -405.708972 & 3.15 & 4.24 & 1.09 \\
6-311++G(d,p) & -405.679848 & 3.07 & 4.30 & 1.23 \\
6-311+G(d,p) & -405.679675 & 3.07 & 4.29 & 1.22 \\
6-311G(d,p) & -405.674322 & 2.97 & 5.50 & 2.53 \\
6-31++G(d,p) & -405.594573 & 3.17 & 4.21 & 1.04 \\
6-31+G(d,p) & -405.594306 & 3.20 & 4.23 & 1.03 \\
6-31G(d,p) & -405.580069 & 2.76 & 5.84 & 3.08 \\
HF & & & & \\
cc-pVTZ & -403.056920 & 3.75 & 4.33 & 0.58 \\
6-311++G(3df,2pd) & -403.049900 & 3.78 & 4.36 & 0.58 \\
6-311++G(d,p) & -403.015421 & 3.64 & 4.42 & 0.78 \\
6-311+G(d,p) & -403.015159 & 3.66 & 4.41 & 0.75 \\
6-311G(d,p) & -403.010816 & 3.69 & 5.36 & 1.67 \\
6-31++G(d,p) & -402.938265 & 3.74 & 4.32 & 0.59 \\
6-31+G(d,p) & -402.937899 & 3.71 & 4.27 & 0.56 \\
6-31G(d,p) & -402.931247 & 3.59 & 5.53 & 1.94 \\
\hline & & & & \\
\hline
\end{tabular}

${ }^{a} E_{M M P}-E_{O M M}$

${ }^{b}$ No frequency calculation at this level of theory. Therefore, only the electronic contribution without zero-point correction is used. 
Table 3. Molecular parameters of the observed conformers OPM and OMM of DEPA obtained from the fits using the program XIAM (obs.) and from quantum chemical calculations (calc.).

\begin{tabular}{llllll}
\hline Par. & Unit & \multicolumn{2}{c}{ 0PM } & \multicolumn{2}{c}{ 0MM } \\
\hline & & \multicolumn{1}{c}{ obs. $^{\mathrm{a}}$} & \multicolumn{1}{c}{ calc. $^{\mathrm{a}}$} & \multicolumn{1}{c}{ calc. $^{\mathrm{a}}$} \\
\hline$A$ & $\mathrm{MHz}$ & $1924.21584(15)$ & $1915.4^{\mathrm{b}}$ & $2070.06910(24)$ & $2071.2^{\mathrm{b}}$ \\
$B$ & $\mathrm{MHz}$ & $1214.565984(56)$ & $1220.8^{\mathrm{b}}$ & $1180.371868(71)$ & $1177.1^{\mathrm{b}}$ \\
$C$ & $\mathrm{MHz}$ & $832.070891(42)$ & $833.7^{\mathrm{b}}$ & $857.533403(51)$ & $856.3^{\mathrm{b}}$ \\
$D_{J}$ & $\mathrm{kHz}$ & $0.10699(53)$ & $0.1318^{\mathrm{c}}$ & $0.18648(54)$ & $0.1482^{\mathrm{c}}$ \\
$D_{J K}$ & $\mathrm{kHz}$ & $0.6118(26)$ & $0.6399^{\mathrm{c}}$ & $0.3234(30)$ & $0.5969^{\mathrm{c}}$ \\
$D_{K}$ & $\mathrm{kHz}$ & $-0.0874(60)$ & $0.1274^{\mathrm{c}}$ & $0.850(18)$ & $0.4089^{\mathrm{c}}$ \\
$d_{J}$ & $\mathrm{kHz}$ & $-0.06218(32)$ & $-0.0771^{\mathrm{c}}$ & $-0.05280(38)$ & $-0.0434^{\mathrm{c}}$ \\
$d_{K}$ & $\mathrm{kHz}$ & $-0.02384(16)$ & $-0.0260^{\mathrm{c}}$ & $-0.01435(21)$ & $-0.0157^{\mathrm{c}}$ \\
$\chi_{a a}$ & $\mathrm{MHz}$ & $1.97018(79)$ & $1.9490^{\mathrm{d}}$ & $1.79861(93)$ & $1.8180^{\mathrm{d}}$ \\
$\chi_{b b} \chi_{c c}$ & $\mathrm{MHz}$ & $4.2749(14)$ & $4.2432^{\mathrm{e}}$ & $6.6695(15)$ & $6.6103^{\mathrm{e}}$ \\
$\chi_{b b}$ & $\mathrm{MHz}$ & $1.15236(40)^{\mathrm{e}}$ & $1.1471^{\mathrm{d}}$ & $2.43544(47)^{\mathrm{e}}$ & $2.3961^{\mathrm{d}}$ \\
$\chi_{c c}$ & $\mathrm{MHz}$ & $-3.2255(88)^{\mathrm{e}}$ & $-3.0961^{\mathrm{d}}$ & $-4.2340(10)^{\mathrm{e}}$ & $-4.2142^{\mathrm{d}}$ \\
$\sigma^{\mathrm{f}}$ & $\mathrm{kHz}$ & 2.2 & & 1.7 & \\
$\mathrm{~N}_{\mathrm{rot}} / \mathrm{N}_{\mathrm{q}}^{\mathrm{g}}$ & & $91 / 368$ & & $56 / 220$ & \\
\hline
\end{tabular}

a Parameters are given with one standard uncertainty in parentheses. Watson's S reduction and $\mathrm{I}^{\mathrm{r}}$ representation were used. $\mathrm{b}$ Obtained from the structure optimized at the MP2/6-311++G(d,p) level of theory. ${ }^{\mathrm{c}}$ Obtained from anharmonic frequency calculation at the same level. ${ }^{\mathrm{d}}$ See the section on NQCCs. ${ }^{\mathrm{e}}$ Derived. ${ }^{\mathrm{f}}$ Standard deviation of the fit. ${ }^{\mathrm{g}}$ Number of rotational $\left(\mathrm{N}_{\text {rot }}\right)$ and hyperfine transitions $\left(\mathrm{N}_{\mathrm{q}}\right)$. 
Table 4. ${ }^{14} \mathrm{~N}$ NQCC $z$-principal axis component $\chi_{z z}$ (in MHz) of DEPA and related amides.

\begin{tabular}{llll}
\hline & & $\chi_{z z}$ & Ref. \\
\hline Formamide & $\mathrm{H}(\mathrm{C}=\mathrm{O}) \mathrm{NH}_{2}$ & $-3.8510(11)$ & {$[21]$} \\
Propionamide & $\mathbf{C H}_{3} \mathbf{C H}_{2}(\mathrm{C}=\mathrm{O}) \mathrm{NH}_{2}$ & $-3.9762(48)$ & {$[22]$} \\
N-Ethylformamide & $\mathrm{H}(\mathrm{C}=\mathrm{O})(\mathrm{NH}) \mathbf{C H}_{2} \mathbf{C H}_{3}$ & $-3.993(51)$ & {$[7]$} \\
N-Methylpropionamide & $\mathbf{C H}_{3} \mathbf{C H}_{2}(\mathrm{C}=\mathrm{O})(\mathrm{NH}) \mathbf{C H}_{3}$ & $-4.168(19)$ & {$[23]$} \\
N-Ethylacetamide & $\mathbf{C H}_{3}(\mathrm{C}=\mathrm{O})(\mathrm{NH}) \mathbf{C H}_{2} \mathbf{C H} 3$ & $-4.118(39)$ & {$[9]$} \\
N,N-Diethylacetamide & $\mathbf{C H}_{3} \mathrm{C}(=\mathrm{O}) \mathrm{N}\left(\mathbf{C H}_{3} \mathbf{C H}_{2}\right)_{2}$ & $-4.389(40) /-4.408(50)$ & {$[9]$} \\
N,N-Diethylpropionamide & $\mathbf{C H}_{3} \mathbf{C H}(\mathbf{C}=\mathrm{O}) \mathrm{N}\left(\mathbf{C H}_{3} \mathbf{C H}_{2}\right)_{2}$ & $-4.455(55)^{\mathrm{a}} /-4.432(34)^{\mathrm{b}}$ & $\mathrm{c}$ \\
\hline
\end{tabular}

${ }^{\text {a }}$ Calculated through diagonalization of the quadrupole tensor with the off diagonal elements $\chi_{a b}=0.243(69), \chi_{a c}=0.544(69)$, and $\chi_{b c}=2.662(69) \mathrm{MHz}$. The estimated uncertainty of the offdiagonal elements is twice the root mean square difference between the calculated and experimental diagonal components. ${ }^{\mathrm{b}}$ Similar to ${ }^{\mathrm{a}}$ with $\chi_{a b}=0.057(97), \chi_{a c}=0.1062(97)$, and $\chi_{b c}=0.336(97) \mathrm{MHz}{ }^{\mathrm{c}}$ This work.

\section{Graphical abstract}

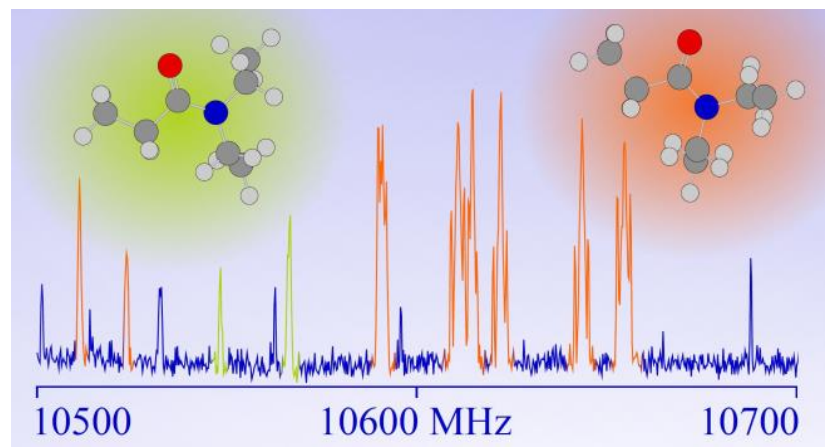

